PROCEEDINGS OF THE

AMERICAN MATHEMATICAL SOCIETY

Volume 126, Number 1, January 1998, Pages 203-211

S 0002-9939(98)04014-3

\title{
THE SCHRÖDINGER OPERATOR
}

\author{
TIAN LIXIN AND LIU ZENGRONG
}

(Communicated by Christopher D. Sogge)

\begin{abstract}
In this paper we study the maximum dissipative extension of the Schrödinger operator, introduce the generalized indefinite metric space, obtain the representation of the maximum dissipative extension of the Schrödinger operator in the natural boundary space and make preparation for the further study of the longtime chaotic behavior of the infinite-dimensional dynamics system in the Schrödinger equation.
\end{abstract}

\section{INTRODUCTION}

The study of longtime chaotic behavior of the nonlinear Schrödinger equation makes physics theory richer. Nonlinear Schrödinger equation problems are difficult since the equation is very complicated. These problems appear in [1]-[4]. In particular, [1] studies the attractors and fractal dimension of the equation. We study the equation by the methods developed recently in studying the longtime behavior of the infinite-dimensional dynamics system. In this paper we will give the representation of the maximum dissipative extension of the Schrödinger operator in the equation. In Section 2, by the generalized semi-inner product space we give a new space called the generalized indefinite metric space, which holds by the meaning of the Banach space in general. We give the natural boundary space of the Schrödinger operator, which is a generalized indefinite metric space. And by the indefinite metric of the natural boundary space, we give the representation of the one-to-one correspondence between the maximum dissipative extension of the Schrödinger operator and the maximum negative subspace of the natural boundary space. In Section 3 we discuss the theorems and give some examples.

According to [4], the Schrödinger operator is $-h \Delta+V$, defined in $C^{\infty}(M)$, where $M$ is a $C^{\infty}$-compact Riemann manifold. The Schrödinger operator has a unique selfadjective extension in Sobolev space $H^{2}(M)$. But if the domain isn't a Riemann manifold, the operator becomes complex and the study of the Schrödinger equation becomes difficult (see [8], [9]). In this paper, we study the operator for the domain in Banach space, and give the maximum dissipative extension of the

Received by the editors February 28, 1996 and, in revised form, July 16, 1996.

1991 Mathematics Subject Classification. Primary 46C50, 47A20, 47B39, 47B44, 81Q05.

Research supported in part by the National Science Foundation of China and Sciencetechnology Foundation of the Ministry of Machine-building Industry of China.

(C)1998 American Mathematical Society 
operator. Suppose

$$
\begin{gathered}
L_{0} f=i f^{\prime \prime}-f, \\
D\left(L_{0}\right)=\left\{f: f, f^{\prime}, f^{\prime \prime} \in L^{p^{\prime}}[0,2 \pi], f(0)=f(2 \pi), f^{\prime}(0)=f^{\prime}(2 \pi), 1<p<\infty\right\}
\end{gathered}
$$

where $L_{0}$ is the type of Schrödinger operator which will be studied. Let $X=$ $L^{p^{\prime}}[0,2 \pi]$, its generalized semi-inner product $[\cdot, \cdot]_{p}$ (see $\left.[5]\right)$, where

$$
[f, g]_{p}=\int_{0}^{2 \pi} f_{g}^{-}|g|^{p-2} d x, \quad 1<p<+\infty
$$

$p$ may be different from $p^{\prime}$. Obviously the norm in $X$ is $\|f\|=[f, f]_{p}^{1 / p}$.

Suppose $A: D\left(L_{0}\right) \rightarrow X, A f=i f^{\prime \prime}$. Let $G\left(L_{0}\right)=\left\{\left\{f, L_{0} f\right\}: f \in D\left(L_{0}\right)\right\}$. In $X \times X$, construct $Q(\cdot, \cdot)$ by

$$
\begin{aligned}
Q(f, g) & =(f g)^{\prime}(2 \pi)-(f g)^{\prime}(0) \\
& =f^{\prime}(2 \pi) g(2 \pi)-f^{\prime}(0) g(0)+f(2 \pi) g^{\prime}(2 \pi)-f(0) g^{\prime}(0) .
\end{aligned}
$$

Let $\bar{H}=\bar{H}_{+} \oplus \bar{H}_{-}$; here

$$
\bar{H}_{+}=\overline{\operatorname{span}}\{f \in X, Q(f, f) \geq 0\}, \quad \bar{H}_{-}=\overline{\operatorname{span}}\{f \in X, Q(f, f) \leq 0\} .
$$

Denote $\widehat{H}=\bar{H} / G\left(L_{0}\right)$. Construct $\widehat{Q}=\widehat{Q}_{+}+\widehat{Q}_{-}$in $\widehat{H}$, where

$$
\begin{gathered}
\widehat{Q}_{+}\left(\hat{f}_{+}, \hat{g}_{+}\right)=Q\left(\hat{f}_{+}, \hat{g}_{+}\right) \operatorname{sign} Q\left(\hat{g}_{+}, \hat{g}_{+}\right), \quad \hat{f}_{+}, \hat{g}_{+} \in \bar{H}_{+}, \\
\widehat{Q}_{-}\left(\hat{f}_{-}, \hat{g}_{-}\right)=Q\left(\hat{f}_{-}, \hat{g}_{-}\right)\left\{-\operatorname{sign} Q\left(\hat{g}_{-}, \hat{g}_{-}\right)\right\}, \quad \hat{f}_{-}, \hat{g}_{-} \in \bar{H}_{-} .
\end{gathered}
$$

Let $\check{H}=\left\{f: f, f^{\prime} \in X\right\}, \widetilde{H}=\widehat{H} \oplus \check{H}$, and for any $\tilde{f} \in\{\hat{f}, \check{f}\}$, define $\widetilde{Q}$ by

$$
\widetilde{Q}(\tilde{f}, \tilde{g})=\widehat{Q}(\hat{f}, \hat{g})+[\check{f}, \check{g}]_{p} .
$$

Theorem 1. A is a symmetric operator (for the definition see [5]) in Banach space $X$.

Theorem 2. $\left(\bar{H}_{+} / G\left(L_{0}\right), \widehat{Q}_{+}\right),\left(\bar{H}_{-} / G\left(L_{0}\right),-\widehat{Q}_{-}\right)$are generalized semi-inner product spaces.

Theorem 3. $(\widehat{H}, \widehat{Q})$ is a generalized indefinite metric space.

Theorem 4. If the maximum dissipative extension of $L_{0}$ is $L$, then $L$ responds one-to-one with the maximum negative subspace $\widetilde{N}$ of $\widetilde{H}$ and

$$
\begin{gathered}
L u=i u^{\prime \prime}-u+\varphi(\hat{u}), \\
D(L)=\left\{u: u, u^{\prime}, u^{\prime \prime} \in X, \hat{u} \in \widehat{N}\right\},
\end{gathered}
$$

where $\widehat{N}$ is theprojection of $\widetilde{N}$ from $\widetilde{H}$ to $\widehat{H}$. 


\section{Proof of the TheOREMS}

In this paper, the operator $L_{0}$ is defined in Banach space and, naturally, the study of the operator $L_{0}$ in Banach space is more difficult than the study of the operator in Hilbert space. For this reason, we construct generalized semi-inner products in Banach space and use a generalized $p$-selfadjoint, dissipative operator (see [5], [10]) in Banach space. The study of the indefinite metric space can be seen in [6] and [7]. Now we construct the generalized indefinite metric space. Further and deeper study of this space will be given in another paper.

Definition 1. $R$ is a complex (or real) linear space. Define $\langle y, z\rangle$ for arbitrary $x, y, z \in R, \lambda \in C$ (complex), if the following conditions are satisfied:

(1) $\langle x+y, z\rangle=\langle x, z\rangle+\langle y, z\rangle,\langle\lambda x, y\rangle=\lambda\langle x, y\rangle$.

(2) For arbitrary $y \in R$, if $\langle x, y\rangle=0$, then $x=0$.

Thus we call $(R,\langle\cdot, \cdot\rangle)$ a generalized Krein space.

Definition 2. The space $(R,\langle\cdot, \cdot\rangle)$ is called a generalized indefinite metric space, if it includes two subspaces $H_{+}, H_{-}$and

(1) $R=H_{+} \oplus H_{-}$, where $\oplus$ is direct sum and orthogonal according to $\langle\cdot, \cdot\rangle$, that is, $\langle x, y\rangle=0, x \in H_{+}, y \in H_{-}$;

(2) $\left(H_{+},\langle\cdot, \cdot\rangle\right),\left(H_{-},-\langle\cdot, \cdot\rangle\right)$ are generalized semi-inner product spaces, where $H_{+}=\{x \in R \mid\langle x, x\rangle \geq 0\}, H_{-}=\{x \in R \mid\langle x, x\rangle \leq 0\}$, and $H_{+}$and $H_{-}$are called the positive subspace and negative subspace, respectively.

Lemma 1. $R$ is a generalized indefinite metric space. If $x, y \in R$, then $x=x_{+}+x_{-}$ and $y=y_{+}+y_{-}$, where $x_{+}, y_{+} \in H_{+}, x_{-}, y_{-} \in H_{-}$. Let $[x, y]=\left\langle x_{+}, y_{+}\right\rangle+\left\langle x_{-}, y_{-}\right\rangle$; then $(R,[\cdot, \cdot])$ is a generalized semi-inner product space.

Proof. The main point is to prove the inequality

$$
|[x, y]| \leq[x, x]^{1 / p}[y, y]^{(p-1) / p}, \quad p>1 .
$$

Since $a b \leq a^{p} / p+b^{q} / q, a>0, b>0$, where $1 / p+1 / q=1$, it is sufficient to prove

$$
\left(1+k m^{p-1}\right)^{p} \leq\left(1+k^{p}\right)\left(1+m^{p}\right)^{p-1} .
$$

The inequality is proven by using a series of Young inequalities. We omit its proof.

Define projections $P_{ \pm}: \Pi=(R,\langle\cdot, \cdot\rangle) \rightarrow H_{ \pm}$, so that $x=x_{+}+x_{-} \in \Pi \rightarrow x_{ \pm} \in$ $H_{ \pm}$.

Lemma 2. The necessary and sufficient condition for the nonnegative subspace $L$ to be a maximum nonnegative subspace is that $P_{+} L=H_{+}$. Every nonnegative subspace is included in a maximum nonnegative subspace.

We omit the proof. 
Proof of Theorem 1. Assume $f, g \in D\left(L_{0}\right)$; then

$$
\begin{aligned}
{[A f, g]_{p} } & =\int_{0}^{2 \pi} i f^{\prime \prime} \bar{g}|g|^{p-2} d x=\int_{0}^{2 \pi} i f^{\prime \prime} \bar{g}\left((p-2) \int_{0}^{|g|} \alpha^{p-3} d \alpha\right) d x \\
& =\int_{0}^{2 \pi} i f^{\prime \prime} \bar{g}(p-2)\left(\int_{0}^{\infty} X_{[0,|g|]}(x) \alpha^{p-3} d \alpha\right) d x \\
& =(p-2) \int_{0}^{\infty} \alpha^{p-3}\left(\int_{0}^{2 \pi} i \bar{g} X_{[|g|>\alpha]}(x) d f^{\prime}\right) d \alpha \\
& =(p-2) \int_{0}^{\infty} \alpha^{p-3}\left(-\int_{0}^{2 \pi} i \bar{g}^{\prime} X_{[|g|>\alpha]}(x) d f\right) d \alpha \\
& =(p-2) \int_{0}^{\infty} \alpha^{p-3} \int_{0}^{2 \pi} f \overline{\left(-i g^{\prime \prime}\right)} X_{[|g|>\alpha]}(x) d x d \alpha \\
& =(p-2) \int_{0}^{\infty} \alpha^{p-3} \int_{0}^{2 \pi} f \overline{i g^{\prime \prime}} X_{\left[\left|g^{\prime \prime}\right|>\alpha\right]}(x) d x d \alpha \\
& =-\int_{0}^{2 \pi} f \overline{\left(i g^{\prime \prime}\right)} \int_{0}^{\left|g^{\prime \prime}\right|} d\left(\alpha^{p-2}\right) d x=-\int_{0}^{2 \pi} f \overline{i g^{\prime \prime}}\left|g^{\prime \prime}\right|^{p-2} d x=-[f, A g]_{p} .
\end{aligned}
$$

Hence $A$ is a symmetric operator.

Proof of Theorem 2. Since $\bar{f} \in G\left(L_{0}\right)$, it follows that $\bar{Q}_{ \pm}(\bar{f}, \bar{f})=0$ and $G\left(L_{0}\right)$ is a kernel space.

Now to prove that $\bar{Q}_{+}(\cdot, \cdot)$ is a generalized semi-inner product space, we only need to prove

$$
\left|\bar{Q}_{+}(\bar{f}, \bar{g})\right| \leq\left|\bar{Q}_{+}(\bar{f}, \bar{f})\right|^{1 / p}\left|\bar{Q}_{+}(\bar{g}, \bar{g})\right|^{(p-1) / p}, \quad \bar{f}, \bar{g} \in \bar{H}_{ \pm} .
$$

In fact,

$$
\left|\bar{Q}_{+}(\bar{f}, \bar{g})\right|=\left|\operatorname{Re}\left[A^{*} f, g\right]_{p}\right|=0.5\left|\operatorname{Re}\left[A^{*} f, g\right]_{p}+\operatorname{Re}\left[f, A^{* *} g\right]_{p}\right| .
$$

Since $A^{*} \supset-A,-A^{* *} \subset A^{*}$, then

$$
\left|\bar{Q}_{+}(\bar{f}, \bar{g})\right|=0.5\left|\operatorname{Re}\left[A^{*} f, g\right]_{p}+\operatorname{Re}\left[f,-A^{*} g\right]_{p}\right| .
$$

Construct a new generalized semi-inner product in $H \times H$ using that

$$
[\bar{u}, \bar{v}]_{12}=\operatorname{Re}\left[u^{1}, v^{1}\right]_{p}+\operatorname{Re}\left[u^{2}, v^{2}\right]_{p}, \quad \bar{u}=\left\{u^{1}, u^{2}\right\}, \bar{v}=\left\{v^{1}, v^{2}\right\} \in H \times H .
$$

Similarly to Lemma $1,[\cdot, \cdot]_{12}$ is a generalized semi-inner product in $H \times H$. Let

$$
W \bar{u}=W\left\{u^{2}, u^{1}\right\}=\left\{u^{1}, u^{2}\right\}, \quad \bar{u} \in H \times H .
$$

Then $W$ is a generalized $p$-selfadjoint operator in $\left(H \times H,[\cdot, \cdot]_{12}\right)$. Let

$$
W_{1} \bar{u}=W \bar{u}, \quad \bar{u} \in H_{+} ; \quad W_{1} \bar{u}=-W \bar{u}, \quad \bar{u} \in \bar{H}_{+} \backslash H_{+},
$$

$W_{1}$ is a generalized positive operator (see [5]); we easily prove the inequality

$$
\left|\left[W_{1} \bar{u}, \bar{v}\right]_{12}\right| \leq\left|\left[W_{1} \bar{u}, \bar{u}\right]_{12}\right|^{1 / p}\left|\left[W_{1} \bar{v}, \bar{v}\right]_{12}\right|^{(p-1) / p},
$$


and that

$$
\begin{aligned}
\left|\bar{Q}_{+}(\bar{f}, \bar{g})\right| \leq & 0.5\left|\left[W u^{\prime}, u^{\prime}\right]_{12}\right|^{1 / p}\left|\left[W v^{\prime}, v^{\prime}\right]_{12}\right|^{(p-1) / p} \\
= & 0.5\left\{\left|\operatorname{Re}\left[A^{*} f, f\right]_{p}+\operatorname{Re}\left[u, A^{* *} f\right]_{p}\right|\right\}^{1 / p} \\
& \times\left\{\left|\operatorname{Re}\left[A^{*} g, g\right]_{p}+\operatorname{Re}\left[g, A^{* *} g\right]_{p}\right|\right\}^{(p-1) / p} \\
= & \left|\bar{Q}_{+}(\bar{f}, \bar{f})\right|^{1 / p}\left|\bar{Q}_{+}(\bar{g}, \bar{g})\right|^{(p-1) / p}
\end{aligned}
$$

where $u^{\prime}=\left\{f, A^{*} f\right\}, v^{\prime}=\left\{g, A^{*} g\right\}$. This completes the proof.

Lemma 3. $\widehat{Q}(\hat{f}, \hat{g})=\bar{Q}(\bar{f}, \bar{g})$; here $\hat{f}, \hat{g}$ are cosets of $\bar{f}, \bar{g}$, where $\hat{f}, \hat{g} \in \widehat{H}$ and $\bar{f}, \bar{g} \in \bar{H}$.

Proof. Obviously $\widehat{Q}(\hat{f}, \hat{f})=0$, when $\hat{f} \in G\left(L_{0}\right)$. Since $\hat{f} \in \widehat{H}$ and $\bar{f}_{0} \in G\left(L_{0}\right)$, first we prove that $\widehat{Q}\left(\hat{f}+\bar{f}_{0}, \hat{f}\right)=\widehat{Q}(\hat{f}, \hat{f})$. In fact

$$
\widehat{Q}\left(\hat{f}+\bar{f}_{0}, \hat{f}\right)=\bar{Q}_{+}\left(\bar{f}_{+}+\bar{f}_{0}, \bar{f}_{+}\right)+\bar{Q}_{-}\left(\bar{f}_{-}, \bar{f}_{-}\right),
$$

$$
\begin{aligned}
\bar{Q}_{+}\left(\bar{f}_{+}+\bar{f}_{0}, \bar{f}_{+}\right) & =Q\left(\bar{f}_{+}+\bar{f}_{0}, \bar{f}_{+}\right) \operatorname{sign} Q\left(\bar{f}_{+}, \bar{f}_{+}\right) \\
& =\left[A^{*}\left(f_{+}+f_{0}\right), f_{+}\right]_{p} \operatorname{sign} Q\left(\bar{f}_{+}, \bar{f}_{+}\right) \\
& =\left[A^{*} f_{+}, f_{+}\right]_{p} \operatorname{sign} Q\left(\bar{f}_{+}, \bar{f}_{+}\right)+\left[A f_{0}, f_{+}\right]_{p} \operatorname{sign} Q\left(\bar{f}_{+}, \bar{f}_{+}\right) \\
& =\bar{Q}_{+}\left(\bar{f}_{+}, \bar{f}_{+}\right)+\left[A f_{0}, f_{+}\right]_{p} \operatorname{sign} Q_{-}\left(\bar{f}_{+}, \bar{f}_{+}\right),
\end{aligned}
$$

where $\bar{f}_{+}=\left\{f_{+}, L_{1} f_{+}\right\} \in \bar{H}_{+}, L_{1}=A^{*}-I, \bar{f}_{0}=\left\{f_{0}, L_{0} f_{0}\right\} \in G\left(L_{0}\right)$. Now to prove $\left[A f_{0}, f_{+}\right]=0$.

From Theorem 2, similarly, we have

$$
\begin{aligned}
\left|\left[A f_{0}, f_{+}\right]_{p}\right| & =\left|Q\left(\bar{f}_{0}, \bar{f}_{+}\right)\right|=0.5\left|\left[W f_{0}^{\prime}, f_{+}^{\prime}\right]_{12}\right| \\
& \leq 0.5\left|\left[W f_{0}^{\prime}, f_{0}^{\prime}\right]_{12}\right|^{1 / p}\left|\left[W f_{+}^{\prime}, f_{+}^{\prime}\right]_{12}\right|^{(p-1) / p} \\
& \leq 0.5\left|\left[A f_{0}, f_{0}\right]_{p}\right|^{1 / p}\left|\left[W f_{+}^{\prime}, f_{+}^{\prime}\right]_{12}\right|^{(p-1) / p}=0
\end{aligned}
$$

where $f^{\prime}=\left\{f, A^{*} f\right\}, \bar{f}_{0}=\left\{f_{0}, L_{0} f_{0}\right\}, \bar{f}_{+}=\left\{f_{+}, L_{1} f_{+}\right\}$.

Hence $\left[A f_{0}, f_{+}\right]_{p}=0$ and

$$
\widehat{Q}\left(\hat{f}+\bar{f}_{0}, \hat{f}\right)=\bar{Q}\left(\bar{f}+\bar{f}_{0}, \bar{f}\right)=\bar{Q}_{+}\left(\bar{f}_{+}, \bar{f}_{+}\right)+\bar{Q}_{-}\left(\bar{f}_{-}, \bar{f}_{-}\right)=\bar{Q}(\bar{f}, \bar{f}) .
$$

Now we prove $\widehat{Q}\left(\hat{f}, \hat{f}+\bar{f}_{0}\right)=\bar{Q}\left(\bar{f}, \bar{f}+\bar{f}_{0}\right)=\bar{Q}(\bar{f}, \bar{f}), \bar{f} \in \bar{H}_{+} \oplus \bar{H}_{-}, \bar{f}_{0} \in G\left(L_{0}\right)$, where $\bar{f}=\bar{f}_{+}+\bar{f}_{-}$. Remark that $\bar{f}_{+}+\bar{f}_{0} \in \bar{H}_{+}, \bar{f}_{-}+\bar{f}_{0} \in \bar{H}_{-}$.

$\bar{Q}\left(\bar{f}, \bar{f}+\bar{f}_{0}\right)=\bar{Q}_{+}\left(\bar{f}_{+}, \bar{f}_{+}+\bar{f}_{0}\right)+\bar{Q}_{-}\left(\bar{f}_{-}, \bar{f}_{-}\right)=\bar{Q}_{+}\left(\bar{f}_{+}, \bar{f}_{+}\right)+\bar{Q}_{-}\left(\bar{f}_{-}, \bar{f}_{-}+\bar{f}_{0}\right)$.

Since

$$
\begin{aligned}
\bar{Q}_{+}\left(\bar{f}_{+}, \bar{f}_{+}+\bar{f}_{0}\right) & =\bar{Q}_{+}\left(\bar{f}_{+}, \bar{f}_{+}\right)+\bar{Q}_{-}\left(0, \bar{f}_{0}\right)=\bar{Q}_{+}\left(\bar{f}_{+}, \bar{f}_{+}\right) \\
& =\bar{Q}_{+}\left(\bar{f}_{+}, \bar{f}_{+}+\bar{f}_{0}\right)=\bar{Q}\left(\bar{f}_{+}, \bar{f}_{+}+\bar{f}_{0}\right),
\end{aligned}
$$

it follows that $\bar{Q}_{+}\left(\bar{f}_{+}, \bar{f}_{+}+\bar{f}_{0}\right)=\bar{Q}_{+}\left(\bar{f}_{+}, \bar{f}_{+}\right)$.

For the same reason we have $\bar{Q}_{-}\left(\bar{f}_{-}, \bar{f}_{-}+\bar{f}_{0}\right)=\bar{Q}_{-}\left(\bar{f}_{-}, \bar{f}_{-}\right)$. Hence

$$
\bar{Q}\left(\bar{f}, \bar{f}+\bar{f}_{0}\right)=\bar{Q}_{+}\left(\bar{f}_{+}, \bar{f}_{+}\right)+\bar{Q}_{-}\left(\bar{f}_{-}, \bar{f}_{-}\right)=\bar{Q}(\bar{f}, \bar{f}) .
$$

Now we get that $\widehat{Q}(\hat{u}, \hat{u})=\bar{Q}(\bar{u}, \bar{u})$, where $\hat{u} \in \bar{H}_{+} / G\left(L_{0}\right) \oplus \bar{H}_{-} / G\left(L_{0}\right), \bar{u} \in$ $\bar{H}_{+} \oplus \bar{H}_{-}$, and $\hat{u}$ is coset of $\bar{u}$. 
Proof of Theorem 3. As $\bar{Q}$ is a generalized indefinite metric and semi-linear, we have that $(\widehat{H}, \widehat{Q})$ is a generalized indefinite metric space.

Let $\widetilde{N}$ be a negative subspace of $(\widetilde{H}, \widetilde{Q})$ and $\widehat{N}$ a projection from $\widetilde{N}$ to $\widehat{H}$, where $\widetilde{H}=\widehat{H} \oplus \check{H}$. We easily prove that $\widehat{N}$ is a negative subspace of $(\widehat{H}, \widehat{Q})$ and

$$
\|\check{u}\|^{p} \leq-\widehat{Q}(\hat{u}, \hat{u})=-\bar{Q}(\bar{u}, \bar{u}) \leq c\|\hat{u}\|^{p}, \quad \text { for any }\{\hat{u}, \check{u}\} \in \widetilde{N} .
$$

Lemma 4. If $\widetilde{N}$ is a maximum negative subspace, then $\widehat{N}$ is a maximum negative subspace of $(\widehat{H}, \widehat{Q})$.

Lemma 5. If $\widehat{N}$ is a maximum negative subspace, then

$$
\|\check{f}\|^{p} \leq-\widehat{Q}(\hat{f}, \hat{f}), \quad \tilde{f}=\{\hat{f}, \check{f}\} \in \tilde{N} .
$$

Define $\varphi: \hat{f} \rightarrow \check{f}$; then $\varphi$ is a contraction mapping from $\widehat{N}$ to $\check{H}$. Also, if $\varphi$ is a contraction mapping, then the graph of $\varphi$ is a maximum negative subspace in $\widetilde{H}$.

Proof of Theorem 4. Assume that $L$ is the maximum dissipative extension; then $\operatorname{Re}[L f, f]_{p} \leq 0, f \in D(L)$. If $f \in D\left(L_{0}\right), g \in D(L)$, then

$$
\begin{gathered}
\operatorname{Re}\left[L_{0} f, g\right]_{p}=\operatorname{Re}[A f, g]_{p}-[f, g]_{p}, \\
\left|\operatorname{Re}[A f, g]_{p}\right| \leq\left|\operatorname{Re}\left[L_{0} f, g\right]_{p}\right|+\left|[f, g]_{p}\right| \leq\left(\left\|L_{0} f\right\|+\|f\|\right)\|g\|^{p-1}, \\
\left|[A f, g]_{p}\right| \leq 2\left(\left\|L_{0} f\right\|+\|f\|\right)\|g\|^{p-1} .
\end{gathered}
$$

Hence, $g \in D\left(A^{*}\right), D\left(L_{1}\right) \supset D(L)$.

For arbitrary $g \in D\left(L_{0}\right), f \in D(L)$, we have

$$
\left|\left[g, L f-L_{1} f\right]_{p}\right| \leq\|g\|\left\|L f-L_{1} f\right\|^{p-1}
$$

From the Riesz representation theorem of the generalized semi-inner product space (see [5]), there exists a unique $\check{f} \in \check{H}$, so that, for any $g \in D\left(L_{0}\right)$,

$$
\left[g, L f-L_{1} f\right]_{p}=[g, f]_{p}, \quad L f-L_{1} f=\check{f} .
$$

Therefore $L u=L_{1} u+\check{f}$.

Let $\widehat{Q}(\hat{f}, \hat{g})=\bar{Q}(\bar{f}, \bar{g})=\bar{Q}_{+}\left(\bar{f}_{+}, \bar{g}_{+}\right)+\bar{Q}_{-}\left(\bar{f}_{-}, \bar{g}_{-}\right)$, where $\hat{f}, \hat{g} \in \widehat{H}$ are cosets of $\bar{f}, \bar{g}$ and $\bar{f}=\bar{f}_{+}+\bar{g}_{-}, \bar{g}=\bar{g}_{+}+\bar{g}_{-}, \bar{f}_{+}, \bar{g}_{+} \in \bar{H}_{+}, \bar{f}_{-}, \bar{g}_{-} \in \bar{H}_{-}$. The following inequality holds:

$$
\widehat{Q}(\hat{f}, \hat{f})-m\|f-\check{f}\|^{p}+\|\check{f}\|^{p} \leq 0 ;
$$

here $m$ is an arbitrary constant, $\bar{u}=\left\{u, L_{1} u\right\} \in G\left(L_{0}\right), \hat{u}$ is coset of $\bar{u}$. First, we prove

$$
Q(\bar{f}, \bar{f})-m\|f-\check{f}\|^{p}+\|\check{f}\|^{p} \leq 0, \quad \bar{f} \in G\left(L_{1}\right) .
$$

Since $Q(\bar{f}, \bar{f})=\operatorname{Re}\left[L_{1} f, f\right]_{p}-[f, f]_{p}$, it follows that

$$
\operatorname{Re}\left[L_{1} f, f\right]_{p}=Q(\bar{f}, \bar{f})-[f, f]_{p} .
$$

As $\operatorname{Re}[L f, f]_{p} \leq 0$ and $\operatorname{Re}\left[L_{1} f+\check{f}, f\right]_{p} \leq 0$,

$$
Q(\bar{f}, \bar{f})-\operatorname{Re}[f, f]_{p}+\operatorname{Re}[\check{f}, f]_{p} \leq 0 .
$$

Hence, to prove (3), we only have to prove

$$
-m\|f-\check{f}\|^{p}+\|\check{f}\|^{p} \leq-[f, f]_{p}+\operatorname{Re}[\check{f}, f]_{p} .
$$


In fact

$$
\begin{aligned}
& \operatorname{Re}[-f+\check{f}, f]_{p} \leq\left|[-f+\check{f}, f]_{p}\right| \leq\|f-\check{f}\|\|f\|^{p-1} \\
& \quad \leq\|f-\check{f}\|^{p} / p+\|f\|^{q(p-1)} / q=\|f-\check{f}\|^{p} / p+\|f\|^{p} / q, \quad 1 / p+1 / q=1 .
\end{aligned}
$$

The left hand side of the above equality is equal to $-[f, f]_{p}+\operatorname{Re}[\check{f}, f]_{p}$. So

$$
[f, f]_{p}-\operatorname{Re}[\check{f}, f]_{p} \geq-\|f-\check{f}\|^{p} / p=\|f\|^{p} / q .
$$

Using the above inequality in (4), we have to prove

$$
\begin{aligned}
& -m\|f-\check{f}\|^{p}+\|\check{f}\|^{p}-\|f-\check{f}\|^{p} / p-\|f\|^{p} / q \leq 0 \\
& \text { or }(-m-1 / p)\|f-\check{f}\|^{p}+\|\check{f}\|^{p} \leq\|f\|^{p} / q .
\end{aligned}
$$

Note that

$$
\begin{aligned}
\operatorname{Re}[-f+\check{f}, \check{f}]_{p} \leq & \left|[-f+\check{f}, \check{f}]_{p}\right| \leq\|-f+\check{f}\|\|\check{f}\|^{p-1} \leq\|f-\check{f}\|^{p} / p+\|\check{f}\|^{p} / q, \\
& q \operatorname{Re}[-f+\check{f}, \check{f}]_{p} \leq q\|f-\check{f}\|^{p} / p+\|\check{f}\|^{p}, \\
& -q \operatorname{Re}[f, \check{f}]+(q-1)\|\check{f}\|^{p} \leq q\|f-\check{f}\|^{p} / p, \\
& -\frac{q}{q-1} \operatorname{Re}[f, \check{f}]_{p}+\|\check{f}\|^{p} \leq \frac{q}{p(q-1)}\|f-\check{f}\|^{p}, \\
& -\frac{q}{q-1} \operatorname{Re}[f, \check{f}]_{p} \leq \frac{q}{p(q-1)}\|f-\check{f}\|^{p}-\|\check{f}\|^{p} .
\end{aligned}
$$

Hence

$$
\begin{aligned}
\|\check{f}\|^{p}-\|f-\check{f}\|^{p} & \leq p \operatorname{Re}[f, \check{f}] \leq p\|f\| \check{f} \|^{p-1} \leq p\left[(l / p)\|f\|^{p}+(1 / q l)\|\check{f}\|^{(p-1) q}\right] \\
& =l\|f\|^{p}+(p / q l)\|\check{f}\|^{p} .
\end{aligned}
$$

Let $l>0$ and $1-p / q l>0$. Take $0<l<(\sqrt{1+4 p}+1) / q$; then $l^{2}-l / q-p / q^{2}<0$, $1-p / q l>0$. By simplifying, we get

$$
(1-p / q l)\|\check{f}\|^{p}-\|f-\check{f}\|^{p} \leq l\|f\|^{p}
$$

or

$$
\|\check{f}\|^{p}-\|f-\check{f}\|^{p} /\left[1-p(q l)^{-1}\right] \leq l\|f\|^{p} /\left[1-p(q l)^{-1}\right] .
$$

Using it in (5), we only have to prove

$$
\left(-m-\frac{1}{p}+\frac{1}{1-p(q l)^{-1}}\right)\|f-\check{f}\|^{p} \leq\left(-\frac{l}{1-p(q l)^{-1}}+\frac{1}{q}\right)\|f\|^{p} .
$$

Take $m=-\frac{1}{p}+\frac{1}{1-p(q l)^{-1}}<0$. At this time, the left hand side of $(6)$ is 0 , and the coefficient of the right hand side is equal to $\frac{l}{1-p(q l)^{-1}}+\frac{1}{q}>0$. Therefore, (6) holds naturally. Hence (3) holds. So

$$
Q(\bar{f}, \bar{f})-m\|f-\check{f}\|^{p}+\|\check{f}\|^{p} \leq 0, \quad \bar{f}=\left\{f, L_{1} f\right\}, m<0 .
$$

It follows that $Q(\bar{f}, \bar{f}) \leq 0, \bar{f} \in \bar{H}_{-}, \bar{Q}_{-}(\bar{f}, \bar{f})=\bar{Q}_{-}(\bar{f}, \bar{f})$.

So $\widehat{Q}(\hat{f}, \hat{f})=\bar{Q}(\bar{f}, \bar{f})=\bar{Q}_{-}(\bar{f}, \bar{f})$. Hence $(2)$ holds.

Since $L v=L_{1} v=L_{0} v, v \in D\left(L_{0}\right), \check{f}$ depends on the coset of $\hat{f}$ in $\widehat{H}, \check{f}=\varphi(\hat{f})$.

Note that $-m<0$, so $-m\|f-\check{f}\|^{p} \geq 0$. Hence, the middle term of equality (2) may be omitted and

$$
\widehat{Q}(\hat{f}, \hat{f})+\|\varphi(\hat{f})\|^{p} \leq 0, \quad f \in D\left(L_{0}\right) .
$$


Therefore $\left\{\{\hat{f}, \varphi(\hat{f})\}, f \in D\left(L_{0}\right)\right\}$ forms a negative subspace corresponding with $\widetilde{Q}$ in $\widetilde{H}$.

On the other hand if $L u=i u^{\prime \prime}-u+\varphi(\hat{u}), L$ is the extension of $L_{0}$ and $\{\{\hat{u}, \varphi(\hat{u})\} \mid u \in D(L)\}$ is the maximum negative subspace of $\widetilde{H}$, then it can be shown that (2) holds, so $L$ is a dissipative operator in Banach space $L^{p}[0,2 \pi]$.

Therefore, there exists a one-to-one correspondence negative subspace of $\widetilde{H}$ and the representation is obtained. This completes the proof.

\section{Discussion}

(1) $L^{p}[0,2 \pi]$ is a typical example of a generalized semi-inner product space (see [5]). The operators of a generalized semi-inner product space are more complicated. In this space there are many operators, for example, generalized $p$-selfadjoint operators [5], symmetric operators [5], dissipative operators [10]. In this paper $L_{0}$ is the dissipative operator in $L^{p}[0,2 \pi]$. In fact,

$$
\begin{aligned}
\operatorname{Re}\left[L_{0} u, u\right]_{p} & =\operatorname{Re}\left[i u^{\prime \prime}-u, u\right]_{p}=\operatorname{Re}[A u, u]_{p}-[u, u]_{p}=-\operatorname{Re}[u, A u]_{p}-[u, u]_{p} \\
& =-\|A u\|^{2-p} \operatorname{Re}[u, A u]_{2}-[u, u]_{p} \\
& =-\|A u\|^{2-p} \operatorname{Re} \int_{0}^{2 \pi} u \overline{i u^{\prime \prime}} d x-[u, u]_{p}=-[u, u]_{p} \leq 0 .
\end{aligned}
$$

Hence $\operatorname{Re}\left[L_{0} u, u\right]_{p} \leq 0$, where $L_{0}$ is a dissipative operator in Banach space $L^{p}[0,2 \pi]$.

Because there doesn't exist an inner product in Banach space, the study of operators in Banach space is difficult and complicated. If $A$ is a selfadjoint operator in Hilbert space $H$, then $(A x, y)=(x, A y)$ and $(A x, x)$ is real, where $(\cdot, \cdot)$ is an inner product in $H$ and $x, y \in H$. But the following example explains that the generalized $p$-selfadjoint operator in Banach space which satisfies $[A x, x]_{p}$ is complex.

Example. $X=Y \oplus Y$, where $\oplus$ is $l_{3}$-direct sum and $Y$ is a two-dimensional Hilbert space. Define the generalized semi-inner product following that, for $1<p<+\infty$,

$$
\left[\left\langle y_{1}, y_{2}\right\rangle,\left\langle y_{1}^{\prime}, y_{2}^{\prime}\right\rangle\right]_{p}=\left\|\left\langle y_{1}^{\prime}, y_{2}^{\prime}\right\rangle\right\|^{p-3}\left\{\left(y_{1}, y_{1}^{\prime}\right)\left\|y_{1}\right\|+\left(y_{2}, y_{2}^{\prime}\right)\left\|y_{2}^{\prime}\right\|\right\}
$$

where $(\cdot, \cdot)$ is an inner product in $Y, x=\left\langle y_{1}, y_{2}\right\rangle, y=\left\langle y_{1}^{\prime}, y_{2}^{\prime}\right\rangle \in X$. Define the operator

$$
T=\left(\begin{array}{cccc}
0 & 0 & -i & 0 \\
0 & 0 & 0 & i \\
i & 0 & 0 & 0 \\
0 & -i & 0 & 0
\end{array}\right)
$$

Easily prove that $[T x, y]_{p}=[x, T y]_{p}$, for arbitrary $x, y \in X$. Thus $T$ is a generalized $p$-selfadjoint operator, but

$$
\begin{aligned}
& x\left[\left\langle y_{1}, y_{2}\right\rangle,\left\langle y_{1}, y_{2}\right\rangle\right]_{p} \\
& \quad=\left\|\left\langle y_{1}, y_{2}\right\rangle\right\|^{p-3}\left\{\left(i x_{21} \bar{x}_{11}-i x_{22} \bar{x}_{12}\right)\left\|y_{1}\right\|+\left(-i x_{11} \bar{x}_{21}+i x_{12} \bar{x}_{22}\right)\left\|y_{2}\right\|\right\},
\end{aligned}
$$

where $y_{1}=\left\langle\left\langle x_{11}, x_{12}\right\rangle\right\rangle, y_{2}=\left\langle\left\langle x_{21}, x_{22}\right\rangle\right\rangle \in H$. Then $[T x, x]_{p}$ is complex, not real.

The generalized indefinite metric space is first introduced in this paper. The discussion and research of new space will be given by another paper. If we define $\left\langle\left\langle\left\langle y_{1}, y_{2}\right\rangle,\left\langle y_{1}^{\prime}, y_{2}^{\prime}\right\rangle\right\rangle\right\rangle_{p}=\left\|\left\langle y_{1}^{\prime}, y_{2}^{\prime}\right\rangle\right\|^{p-3}\left\{\left(y_{1}, y_{1}^{\prime}\right)\left\|y_{1}^{\prime}\right\|-\left(y_{2}, y_{2}^{\prime}\right)\left\|y_{2}^{\prime}\right\|\right\}$, then $\langle\langle\cdot, \cdot\rangle\rangle$ is a generalized indefinite metric in $X$ of the example.

(2) Dissipative operators are playing an increasingly important role as research on nonselfadjoint operators proceeds. Many interesting initial value problems in 
partial differential equations are defined in Banach space. In this paper, we consider this case and study the maximum dissipative extension representation of dissipative operators for the space $L^{p}$ in the Schrödinger equation. The Schrödinger operator $-h \Delta+V($ or $i \Delta-i V)$ is considered, where $V(x)$ is the potential. If $V(x)$ does not satisfy the $L^{2}$-integral, but the $L^{p}$-integral (see [8], [9]), then the particles in the Schrödinger equation could cause colliding and scattering, especially, for $i \Delta-i V$, where $V(x)$ is a complex function in $L^{p}[0,2 \pi]$; particles cause the scattering. It is difficult to study the scattering in quantum mechanics at present. In this paper we try to study one of the Schrödinger operators in $L^{p}[0,2 \pi]$ when $V(x)$ is an imaginary number. There may be a new method of studying the scattering of the Schrödinger equation, but we don't know how the maximum dissipative extension representation of the Schrödinger operator and the scattering of Schrödinger equation connect.

\section{REFERENCES}

1. Yin Yan, Attractors and dimensions for discretizations of a weakly damped Schrödinger equation and a sine-Gordon equation, Nonlinear Analysis 20 (1993), 1417-1452. MR 94k:35303

2. A. Soffer and M. I. Weinstein, Multichannel nonlinear scattering for nonintegrable equations. II, J. Diff. Eq. 98 (1992), 376-390.

3. Nakao Hayashi, The initial value problem for the derivative nonlinear Schrödinger equation in the energy space, Nonlinear Analysis 20 (1993), 823-833. MR 94c:35007

4. B. Helffer, Semi-classical analysis for the Schrödinger operator and applications, Lecture Notes in Math., vol. 1336, Springer-Verlag, New York, 1988. MR 90c:81043

5. Wei Gouqing and Shen Youqiang, The generalized $p$ normal operators and p hyponormal operators on Banach spaces, Chin. Ann. Math. Ser. B 8 (1987), 70-79. MR 88f:47018

6. H. Langer, Spectral functions of definitizable operators in Krein spaces, 1-46, Lecture Notes in Math., vol. 948, Springer-Verlag, New York, 1982. MR 84g:47034

7. L. Bonger, Indefinite inner product spaces, Springer-Verlag, New York, 1974.

8. Peder A. Olsen, Fractional integration, Morrey spaces and a Schrödinger equation, Comm. Part. Diff. Eq. 20 (1995), 2005-2055. MR 97a:35042

9. M. Hoffmann-Ostenhof, T. Hoffmann-Ostenhoff and N. Nadirashvili, Interior Hölder estimates for solutions of Schrödinger equations and the regularity of nodal sets, Comm. Part. Diff. Eq. 20 (1995), 1241-1273. MR 97a:35041

10. G. Lumer, R. S. Phillips, Dissipative operators in a Banach space, Pacific J. Math. 11 (1961), 679-698. MR 24:A2248

11. M. G. Crandall and R. S. Phillips, On the extension problem for dissipative operators, J. Funct. Anal. 11 (1968), 147-176. MR 37:6775

Department of Mathematics and Physics, Jiangsu University of Science and Technology, Zhenjiang, Jiangsu, 212013, People's Republic of China

E-mail address: $\operatorname{lgj} @ j$ sust.edu.cn

Department of Mathematics, Suzhou University, Suzhou, Jiangsu, 215006, People's Republic of China 